\title{
Coordination Scheme and Target Tracking for Distributed Model Predictive Control
}

\author{
Quan Min Shao ${ }^{\mathrm{a}, \mathrm{b}}$, Ali Cinar ${ }^{\mathrm{a}, *}$ \\ ${ }^{a}$ Department of Chemical and Biological Engineering, Illinois Institute of Technology, Chicago, IL 60616, \\ USA \\ ${ }^{b}$ Air Products and Chemicals, Allentown, PA 18195, USA
}

\begin{abstract}
Distributed control provides communication between local controllers, which facilitates the achievement of the global objective for the overall control problem. A coordinator is proposed to implement the communication of local controllers. The presence of the coordinator in the control system provides benefits in the setup of the distributed controllers, and also the calculation of target tracking without the requirement for a feasible starting point. The functionality of the coordinator is illustrated in a case study of the Tennessee Eastman challenge problem.
\end{abstract}

Keywords: Distributed control, Subspace identification, Distributed target tracking, Agent-based systems

\section{Introduction}

The implementation of a centralized MPC may be difficult for various reasons such as geographic diversity or computational limitations. Distributed control, an advanced form of decentralized control can overcome these difficulties. In this paper, distributed model predictive control (DMPC) will be used for advanced control and a novel coordination scheme will be proposed. DMPC decomposes the overall system into several partitions, and every partition is controlled by a local MPC. Each local controller has its own objective function. It conducts the optimization and implements the control action to the local section of the process under its jurisdiction. The difference between distributed MPC and totally decentralized MPC is that distributed MPC may have communication and cooperation between the local MPC controllers, which would be very helpful for ensuring the stability and optimality of the process, although at the expense of consuming additional resources.

The fundamental principles of an effective approach for developing linear DMPC, also called feasible cooperative MPC (FC-MPC) is available in the literature $[1,2]$. The key of this iterative approach is to let the local controllers communicate with each other at a specified rate and whenever new optimized input sequences, that guarantee the global stability and

*Corresponding Author: Phone: 1-312-567-3042, fax: 1-312-567-8874, cinar@iit.edu

Preprint submitted to Elsevier

January 19, 2015

(C) 2015. This manuscript version is made available under the Elsevier user license

http://www.elsevier.com/open-access/userlicense/1.0/ 
(sub)optimality are generated. DMPC of nonlinear systems is also an active research area and methods such as nonlinear DMPC based on Lyapunov-based MPC has been proposed [3], which follows similar information exchange mechanism as FC-MPC. Interest in distributed MPC has increased in recent years and various novel techniques and implementations have been reported $[4,5,6,7,8,9,10,11,12,13,14,15,16]$.

Distributed control fits well in the framework of MADCABS (Monitoring, Analysis, Diagnosis, and Control with Agent-Based Systems), a software platform developed at IIT to provide a real-time supervision and control system for distributed and networked processes $[17,18,19,20,21]$. MADCABS incorporates the multi-agent philosophy to make an adaptive, decentralized, hierarchical supervision system (Figure 1). The proposed coordinator and DMPC are implemented in MADCABS. The MPC controllers and coordinator are agents developed to enhance the closed-loop control functionality of MADCABS.

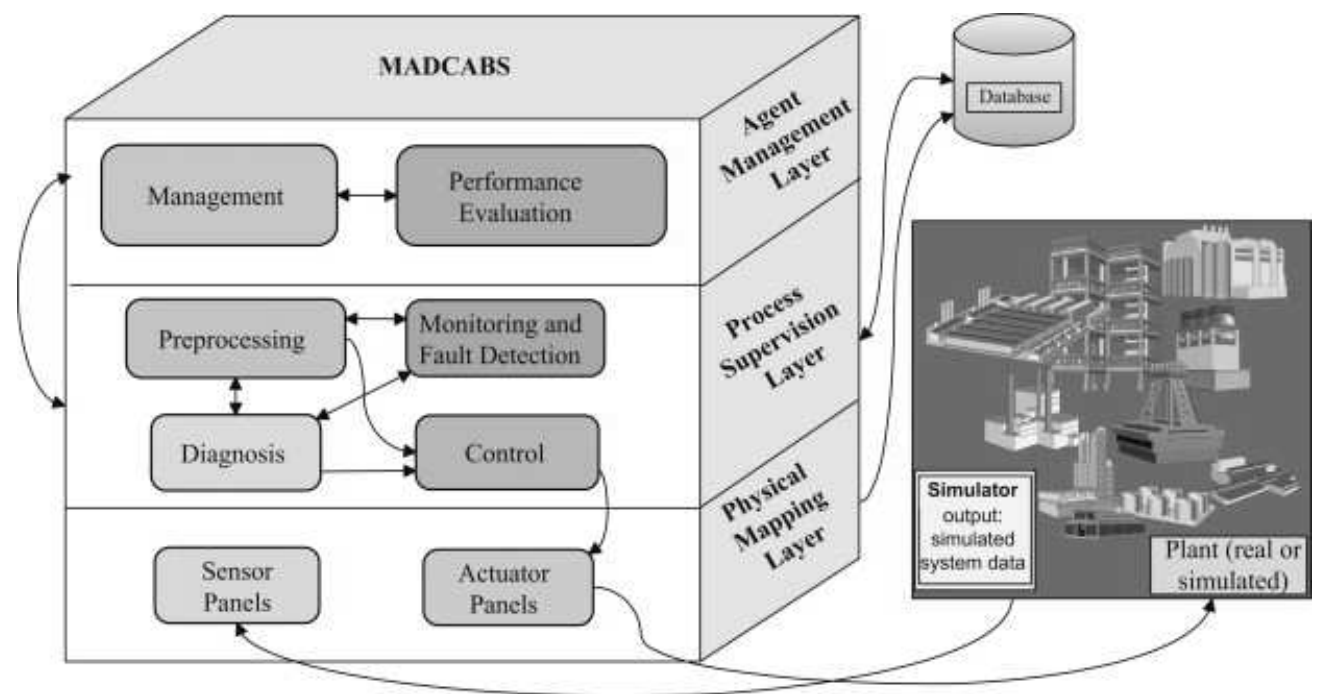

Figure 1: The framework of MADCABS.

In this paper, a coordination scheme is introduced to the iterative DMPC framework. It provides a flexible way to formulate the distributed optimization, which can be updated in a systematic way when local controller is subject to changes. Moreover, an innovative method under coordination scheme is proposed for the calculation of target tracking of DMPC.

The paper is organized as follows. In Section 2, the general linear distributed MPC is introduced. Then a coordination scheme is proposed in Section 3, including two subsections: construction of distributed control optimization and distributed calculation of target tracking. In Section 4, an example is shown for the coordination structure. Conclusions are provided in Section 5. 


\section{Linear Distributed Model Predictive Control}

For a linear system described by the state space model:

$$
\begin{aligned}
x(k+1) & =A x(k)+B u(k) \\
y(k) & =C x(k)
\end{aligned}
$$

The quadratic objective function with prediction horizon $N$ can be written as

$$
\Phi(k)=\sum_{t=k}^{k+N-1}\left(y(t \mid k)^{T} Q y(t \mid k)+u(t \mid k)^{T} R u(t \mid k)\right)+y(k+N \mid k)^{T} \overline{Q_{f}} y(k+N \mid k)
$$

where $Q, R, S, \bar{Q}$ are the weights of outputs, inputs, and the outputs at final stage respectively.

In distributed control, the whole system is divided to subsystems, and each subsystem can consider the inputs from other subsystems. For simplicity, the linear model for subsystem $i$ is described as

$$
\begin{aligned}
x_{i}(k+1) & =A_{i} x_{i}(k)+B_{i} u_{i}(k)+\sum_{j \neq i}^{M} W_{i j} u_{j i}(k) \\
y_{i}(k) & =C_{i} x_{i}(k)
\end{aligned}
$$

where $u_{j i}$ is a subset of $u_{j}$ (the inputs in subsystem $j$ ) and it includes all the inputs that would affect $y_{i}$ (outputs in subsystem $i$ ) in $u_{j}$, and $W_{i j}$ is the corresponding input matrix of $u_{j i}$.

Similar to regular MPC, the local objective function of subsystem $i$ is given in the classical quadratic form

$$
\begin{array}{r}
\Phi_{i}\left(x_{i}(k), \mathbf{u}_{i}\right):=\sum_{j=0}^{N-1}\left(x_{i}(k+j)^{T} C_{i}^{T} Q_{i} C_{i} x_{i}(k+j)+u_{i}(k+j)^{T} R_{i} u_{i}(k+j)\right) \\
+x_{i}(k+N)^{T} C_{i}^{T} \overline{Q_{i}} C_{i} x_{i}(k+N)
\end{array}
$$

where $Q_{i}, R_{i}, S_{i}, \overline{Q_{i}}$ are the weights of outputs, inputs, the change of inputs, and the outputs at final stage for subsystem $i$ respectively.

The overall objective function is the weighted sum of the local objective functions, and the local optimization in subsystem $i$ aims to minimize the overall objective function

$$
\min _{u_{i}} \Phi^{c}=\min _{u_{i}} \sum_{m=1}^{M} w_{m} \Phi_{m}\left(\left[\mathbf{u}_{1}, \ldots, \mathbf{u}_{i-1}, \mathbf{u}_{i}, \mathbf{u}_{i+1}, \ldots, \mathbf{u}_{M}\right] ; x_{m}(k)\right)
$$

by assuming the inputs from other subsystems to be constant. In Equation $5 u_{j}, j \neq i$ is the previous optimization results, which can be from previous iteration or previous time step; $w_{m}$ is the weight with $\sum_{m=1}^{M} w_{m}=1$; and $c$ indicates the centralized variable. There could be multiple iterations in each time step, and the local controllers communicate with each other and update the newly generated optimized inputs sequence $\mathbf{u}_{i}$ after each iteration. It is proved to be stable for any number of iterations It is also proved that when the number of iteration approach infinity, optimality could be achieved the same as centralized MPC [22]. 


\section{Coordination scheme for distributed control}

Instead of letting the controller freely exchange information with each other, a coordinator is utilized in this research. A simple illustration of the coordinator for a system that contains two subsystems is shown in Figure 2. The information flow is only between the coordinator and local controllers. Although the introduction of the coordinator would reduce flexibility and require extra resources, it brings more benefits.

The most straightforward advantage of the coordinator is the simplification of the communications in local controllers: a local controller does not have to keep listening every possible information from other local controllers, instead each local controller only sends information to the coordinator and receives information from the coordinator. Such simplified communication will reduce the risk of problems occurring in complicated communications. In terms of total number of communications, the original structure requires $M(M-1)$ communications, where $M$ is the number of local controllers/subsystems, and the coordination scheme only requires $2 M$ send/receive actions (at the expense of more data transferred in an single communication).

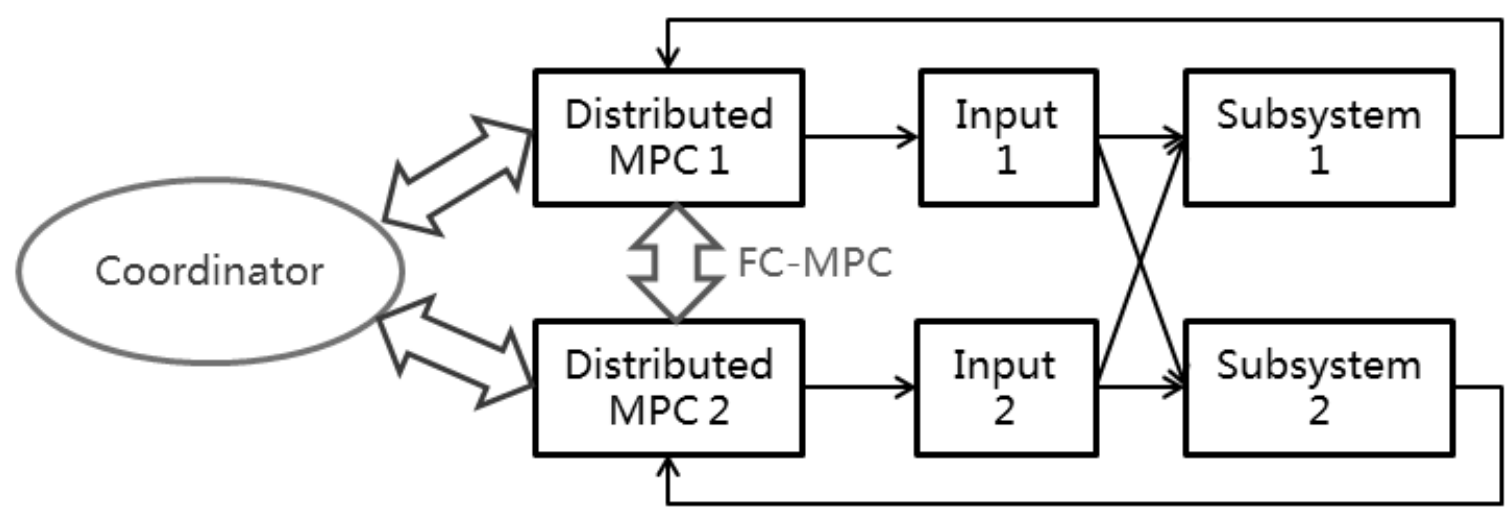

Figure 2: Coordinator in distributed control.

Conceptually, the existence of a coordinator seems contradictory to the idea of distributed systems. If each local controller is represented by an agent in an agent-based framework, the coordinator can be interpreted as another agent in this system, with a different functionally than the other agents representing local controllers. Its computational burden is much less than the other agents (local controllers). The coordinator enables the local controllers to maintain only local information, and the local optimization is constructed by the cooperation between the coordinator and the local controllers, which will be elaborated later in this section.

In this coordination scheme, the coordinator mainly has the following three functionalities

- Management of communication between local controllers.

- Construction of distributed (local) optimization. 
- Calculation of distributed target tracking.

The first functionality has already been presented, and the others will be introduced in the following subsections.

\subsection{Construction of distributed optimization under coordination scheme}

In traditional DMPC design, the distributed optimizations are preassigned to local controllers, which is essentially a centralized procedure. It requires a unit (either a computer or a person) to perform the centralized work, and this unit has the full understanding of the whole process, such as the local model and local objective function of each subsystem. The coordination scheme proposed requires limited centralized information and calculation as well, but it makes the whole system behave in a more distributed way. This is because the coordinator does not have to master the full information, and the information that is required to construct the local optimization problem is gathered from local controllers. In other words, most local knowledge is kept and processed by local controllers, and the coordinator only helps the local controllers achieve the overall objective by making some simple calculations.

The construction of distributed optimization under coordination scheme contains the following three steps:

Step 1 The local controllers construct the local decentralized optimizations as if it is a regular MPC, and send the optimization matrices to the coordinator.

Step 2 The coordinator constructs the centralized optimization matrices by adding together the corresponding elements of local optimization matrices.

Step 3 The coordinator decomposes the centralized optimization to distributed optimization matrices, which is used in the local controllers.

The basic idea of this procedure is to let the local controllers calculate the local objective functions and send them to the coordinator (step 1), then the coordinator sums up the local objective functions to form the overall objective function (step 2), finally the coordinator decomposes the overall objective function and sends them back to the local controllers (step $3)$.

For the first step, first consider the regular MPC with the objective function in Equation 2, subject to the model constraints of Equation 1. The objective function can be converted to the following quadratic form

$$
\Phi(k)=\left(u^{N}(k)\right)^{T} H u^{N}(k)+2\left(u^{N}(k)\right)^{T} G x(k)
$$

Then, it is able to apply the distributed model Equation 3 on the same process, by letting

$$
\begin{aligned}
\beta_{i} & =\left[B_{i}, \overline{B_{i}}\right]=\left[B_{i}, W_{i 1}, \ldots, W_{i(i-1)}, W_{i(i+1)}, \ldots, W_{i N}\right] \\
\mu_{i} & =\left[u_{i}^{T}, \bar{u}_{i}^{T}\right]^{T}=\left[u_{i}^{T}, u_{1 i}^{T}, \ldots, u_{(i-1) i}^{T}, u_{(i+1) i}^{T}, \ldots, u_{N i}^{T}\right]^{T}
\end{aligned}
$$


where $\bar{u}_{i}^{T}$ is the inputs from other subsystems to subsystem $i$, and $\overline{B_{i}}$ includes the corresponding input matrices. Then Equation 3 becomes

$$
x_{i}(k+1)=A_{i} x_{i}(k)+\beta_{i} \mu_{i}(k)
$$

which is applied to local objective function described in Equation 4, and replaces the input weight matrix $R_{i}$ with $\left[\begin{array}{cc}R_{i} & \\ & O_{i}\end{array}\right]$, where $O_{i}$ is a square zero matrix with the number of rows/columns equal the total number of inputs from other subsystems. Then the local objective function can be converted to the following form

$$
\Phi_{i}(k)=\left(\mu_{i}^{N}(k)\right)^{T} H_{i} \mu_{i}^{N}(k)+2\left(\mu_{i}^{N}(k)\right)^{T} G_{i} x_{i}(k)
$$

where the superscript $N$ indicates the sequence from current time to $\mathrm{N}$ time step into the future. In practice, this quadratic programming function can be constructed by the objective function in regular linear quadratic MPC, which is a great advantage for implementation. This step is decentralized, since all calculations are conducted locally, without any interaction with other controllers.

The next step is the computation of the overall objective function on the coordinator side after the local controllers send the decentralized optimization matrices $H_{i}$ and $G_{i}$ to the coordinator. The overall objective is defined in Equation 5, and is restated in an simpler form as below

$$
\Phi^{c}=\sum \omega_{i} \Phi_{i}, \quad \text { with } \quad \sum \omega_{i}=1
$$

In order to realize this summation, define a set $u$ that contains all system inputs, then the input sequence that influences subsystem $i$ can be written as

$$
\mu_{i}^{N}=\Lambda_{i} u^{N}
$$

where $\Lambda_{i}$ is a matrix containing the corresponding rows of the identity matrix. Then the centralized optimization is

$$
\begin{aligned}
u^{N}(k) & =\left[u_{1}^{N}(k)^{T}, \cdots, u_{M}^{N}(k)^{T}\right]^{T} \\
& =\arg \min _{u^{N}} \sum_{i} \omega_{i} \Phi_{i}(k) \\
& =\arg \min _{u^{N}}\left(u^{N}(k)^{T} H^{c} u^{N}(k)+2 u^{N}(k)^{T} G^{c} x(k)\right)
\end{aligned}
$$

where the centralized optimization matrices are

$$
\begin{array}{r}
H^{c}=\sum_{i} \omega_{i} \Lambda_{i}^{T} H_{i} \Lambda_{i} \\
G^{c}=\sum_{i} \omega_{i} \Lambda_{i}^{T} G_{i}
\end{array}
$$


The final step is the decomposition of the centralized optimization results. For subsystem $i$, assume the inputs from other subsystems are known, then the distributed objective function in subsystem $i$ is

$$
\begin{aligned}
\Phi_{i}^{l}(k)= & u_{i}^{N}(k)^{T} \Lambda_{i} H^{c} \Lambda_{i}^{T} u_{i}^{N}(k) \\
& +2 u_{i}^{N}(k)^{T}\left[\Lambda_{i} \frac{1}{2}\left(H^{c}+H^{c T}\right) \bar{\Lambda}_{i}^{T} \overline{u_{i}^{N}}(k)+\Lambda_{i} G^{c} x(k)\right]
\end{aligned}
$$

where $\overline{\Lambda_{i}}$ is the supplementary rows of identity matrix. So far the problem has been formulated as cooperative distributed MPC control. All the constraints are applied to local controller, and the coordinator is only responsible for the formulation of local optimization. The constraints do not affect the decomposition in terms of convergence and optimality, and the stability and (sub)optimality are proved in [1].

This Decentralize - Centralize - Distribute procedure of constructing distributed optimization provides flexibility to build the distributed control system. This coordination scheme essentially enable more autonomy of local controllers. Each local controller is able work on its own, since all the local information (such as models, objective functions, and constraints) are available locally. Meanwhile all the local controllers are able to work together to perform distributed control with the help of the coordinator. Furthermore, local change can be straightforwardly updated to the whole distributed control network via the coordination scheme. This would be particularly useful when the local models require updates occasionally, such as when using recursive system identification for systems undergoing unpredicted disturbances or steady state shifting.

\subsection{Calculation of target tracking under coordination scheme}

Target calculation is challenging in distributed control because the steady state in one subsystem would affect the other subsystems, which is unpredictable within a subsystem locally. One straightforward solution is to make a centralized model for the whole system. However, this approach is against the philosophy of distributed control and it may cause extra computation burden due to the high dimension of the centralized model.

A theoretically sound iterative algorithm has been proposed to solve this problem [22]. It is easy to implement under the framework of distributed control. A shortcoming of this approach is the necessity of a feasible starting point, which is difficult to obtain for the same reason as the optimal target calculation. A new method for the distributed control problem with a linear model is proposed under the new coordination scheme. Although it requires a certain amount of centralized calculation, no starting point is needed, and the calculation is simple on both the distributed controller side and the coordinator side.

A general linear target tracking problem (with or without disturbance model) in subsystem $i$ can be formulated as:

$$
\begin{aligned}
\left(x_{s, i}^{*}, u_{s, i}^{*}\right)= & \arg \min _{x_{s, i}, u_{s, i}} u_{s, i}^{T} R_{s, i} u_{s, i} \\
\text { s.t. } & A_{E, i}\left[\begin{array}{l}
x_{s, i} \\
u_{s, i}
\end{array}\right]=b_{E, i} \\
& u_{s, i} \in \Omega_{i} \\
& 7
\end{aligned}
$$


where subscript $s$ denotes steady state and $*$ denotes optimal solution. The equality constraints include satisfying the process and/or disturbance models at steady state. The idea of the distributed target calculation is based on converting the equality constraints in Equation 15 to the solution space of $A_{E, i}$, which are used as the constraints of centralized optimization for finding a new steady state.

For the distributed system, each subsystem has inputs from the local subsystem and may have inputs from neighbor subsystems. Taking the inputs from other subsystems as arguments to the optimization problem above, the equality constraints are rewritten as:

$$
A_{E, i}\left[\begin{array}{l}
x_{s, i} \\
u_{s, i} \\
\bar{u}_{s, i}
\end{array}\right]=b_{E, i}
$$

where the overline indicates other subsystems and $\overline{u_{s, i}}$ is the vector of inputs from neighbors. The solution space of the equality constraints can be written as:

$$
\left[\begin{array}{l}
u_{s, i} \\
\bar{u}_{s, i}
\end{array}\right]=\left[\begin{array}{l}
u_{p, i} \\
\bar{u}_{p, i}
\end{array}\right]+\left[\begin{array}{c}
V_{i} \\
\bar{V}_{i}
\end{array}\right] \alpha_{i}
$$

where $\alpha_{i}$ is a vector of coefficients, $V_{i}$ is the null space from $A_{E, i}$, and the particular solution $x_{p, i}$ and $u_{p, i}$ can be solved by letting arbitrary $n_{d}$ inputs equal to zero, where $n_{d}$ is the degree of freedom of the linear equation. Then the solution spaces of each subsystem are passed to the centralized coordinator. Index all the inputs in the whole system from 1 to $n_{u}$ as $u^{1}, u^{2}$, $\ldots u^{n_{u}}$, where $n_{u}$ is the total number of inputs. For the $k t h$ input $u^{k}$ in the $i t h$ subsystem, denote $V_{i}^{k}$ and $u_{p, i}^{k}$ as the corresponding row of the matrix of the null space and particular solution, to write

$$
u_{s}^{k}=u_{p, i}^{k}+V_{i}^{k} \alpha_{i}
$$

Then the equality constraints of centralized optimization problem would ensure that $u_{s}^{k}$ from every related subsystem to be equal to each other. Say $u^{k}$ appears in $i t h$ and $j t h$ subsystem, then

$$
u_{p, i}^{k}+V_{i}^{k} \alpha_{i}=u_{p, j}^{k}+V_{j}^{k} \alpha_{j}
$$

And if $u^{k}$ appears in $n$ subsystems, then $n-1$ such constraints are needed to include the equality for $u^{k}$.

When all the local information is collected on the coordinator side, let

$$
\begin{aligned}
R_{s} & =\operatorname{diag}\left(R_{s, 1}, \cdots, R_{s, M}\right) \\
\alpha & =\left[\alpha_{1}^{T}, \cdots, \alpha_{M}^{T}\right]^{T} \\
V & =\operatorname{diag}\left(V_{1}, \cdots, V_{M}\right) \\
u_{p} & =\left[u_{p, 1}{ }^{T}, \cdots, u_{p, M}^{T}\right]^{T}
\end{aligned}
$$

then

$$
u_{s}=u_{p}+V \alpha
$$


Finally, the coefficient $\alpha$ can be obtained from the following optimization:

$$
\begin{aligned}
\alpha^{*}= & \arg \min _{\alpha} \alpha^{T} R_{s}^{\alpha} \alpha+\alpha^{T} f_{s}^{\alpha} \\
\text { s.t. } & A_{E}^{\alpha} \alpha=b_{E}^{\alpha} \\
& u_{p}+V \alpha \in \Omega
\end{aligned}
$$

where

$$
\begin{aligned}
R_{s}^{\alpha} & =V^{T} R_{s} V \\
f_{s}^{\alpha} & =2 V^{T} R_{s} u_{p}
\end{aligned}
$$

and $A_{E}^{\alpha}$ and $b_{E}^{\alpha}$ in the quality matrices can be written as:

$$
\begin{aligned}
& A_{E}^{\alpha}= {\left[\begin{array}{c}
a_{1} \\
\vdots \\
a_{n_{u}}
\end{array}\right], \quad \text { where } a_{k}=\left[\begin{array}{ccc}
a_{1,1}^{k} \cdots & a_{1, M}^{k} \\
\vdots & \ddots & \vdots \\
a_{n_{u}^{k}, 1}^{k} & \cdots & a_{n_{u}^{k}, M}^{k}
\end{array}\right] } \\
& b_{E}^{\alpha}=\left[\begin{array}{c}
b_{1} \\
\vdots \\
b_{n_{u}}
\end{array}\right], \quad \text { where } b_{k}=\left[\begin{array}{c}
b_{1}^{k} \\
\vdots \\
a_{n_{u}^{k}}^{k}
\end{array}\right]
\end{aligned}
$$

and $n_{u}$ is the total number of inputs for the whole system, and $n_{u}^{k}$ is the number of duplicative inputs of the $k t h$ input (e.g. if $u_{1}$ exists in 3 subsystems, then it duplicates twice, so $n_{1}^{k}=2$ ). $a_{k}$ and $b_{k}$ could be set up by the following pseudocode:

$$
\begin{aligned}
& i=\left(\text { the subsystem index that } u_{k} \text { belongs to }\right) \\
& \text { count }=1 \\
& A_{E}^{\alpha}=\mathbf{0}, b_{E}^{\alpha}=\mathbf{0} \\
& \text { for } m \in\left\{\text { the neighbor subsystem indices that } u_{k} \text { affects }\right\} \text { do } \\
& \quad a_{\text {count }, i}^{k}=V_{i}^{k} \\
& \quad a_{\text {count }, m}^{k}=-V_{m}^{k} \\
& \quad b_{\text {count }}^{k}=-u_{s, i}^{k}+u_{s, m}^{k}{ }^{*} \\
& \text { count }++ \\
& \text { end for }
\end{aligned}
$$

It is possible that no feasible solution is available in some tracking problem. In this case, the output target cannot be tracked without offset, and the solution that minimizes the error of output tracking is sought instead. Therefore, the equality constraints $y_{t}-C x_{s}=0$, or $y_{s}-C x_{s}-p=0$ in case of offset-free control with output step disturbance, are relaxed in local controllers. Similar to $u_{s}$, the process states at steady state in the $i t h$ subsystem can be written as:

$$
x_{s, i}=x_{p, i}+V_{x, i} \alpha_{i}
$$


and the new local objective is to minimize the output error at new steady state (constraints are neglected):

$$
\left(x_{s, i}^{*}, u_{s, i}^{*}\right)=\arg \min _{x_{s, i}, e_{y_{s, i}}} e_{y_{s, i}^{T}}^{T} Q_{s, i} e_{y_{s, i}}
$$

where

$$
\begin{aligned}
e_{y, i} & =y_{t, i}-C_{i}\left(x_{p, i}+V_{x, i} \alpha_{i}\right)-p \\
& =z_{i}-\underline{C V_{x}} \alpha_{i}
\end{aligned}
$$

and

$$
\begin{aligned}
z_{i} & =y_{t, i}-C_{i} x_{p, i}-p \\
\underline{C V_{x}} & =C_{i} V_{x, i}
\end{aligned}
$$

Without loss of generality, the output step disturbance term $p$ is included in the above equations, and if no output step disturbance exists, it is set as $p=0$.

Define on the coordinator side:

$$
\begin{aligned}
Q_{s} & =\operatorname{diag}\left(Q_{s, 1}, \cdots, Q_{s, M}\right) \\
\frac{C V_{x}}{\underline{z}} & =\operatorname{diag}\left(\underline{C V_{x_{1}}}, \cdots, \underline{C V_{x}}{ }_{M}\right) \\
z & =\left[z_{1}^{T}, \cdots, z_{M}^{T}\right]^{T}
\end{aligned}
$$

Therefore, the overall output error $e_{y}$ could be written as:

$$
e_{y}=z-\underline{C V_{x}} \alpha
$$

and the target tracking problem becomes the minimization of the quadratic form of $e_{y}$, which could be simplified to:

$$
\begin{aligned}
\alpha^{*}= & \arg \min _{\alpha} e_{y}^{T} Q_{s} e_{y} \\
= & \arg \min _{\alpha} \alpha^{T} Q_{s}^{\alpha} \alpha+\alpha^{T} g_{s}^{\alpha} \\
\text { s.t. } \quad & A_{E}^{\alpha} \alpha=b_{E}^{\alpha} \\
& u_{p}+V \alpha \in \Omega
\end{aligned}
$$

where the constraints are the same as Equation 22, but

$$
\begin{aligned}
Q_{s}^{\alpha} & =\underline{C V_{x}^{T}} Q_{s} \underline{C V_{x}} \\
g_{s}^{\alpha} & =2 \underline{C V_{x}^{T}} Q_{s} z
\end{aligned}
$$

In practice, in addition to the requirement to minimize the output error at the new steady state, it is better and sometimes necessary, to make the new steady state inputs as close to the original operation point as possible (with different weights for different MVs), 
which is the concept of the objective function in feasible case. Therefore a combination of Equations 22 and 31 will be a more effective way to deal with this problem. The new objective function is:

$$
\alpha^{*}=\arg \min _{\alpha} \alpha^{T}\left(Q_{s}^{\alpha}+\beta R_{s}^{\alpha}\right) \alpha+\alpha^{T}\left(g_{s}^{\alpha}+\beta f_{s}^{\alpha}\right)
$$

where $\beta$ is the weight factor, and should be small enough (close to 0 ). This is because the major consideration is still on the output error, while the extra term is to adjust the point in the solution space.

For both feasible and infeasible cases, the final step is to decompose $\alpha^{*}$ and assign $\alpha_{i}^{*}$ to local controllers, where the local optimized inputs and states are constructed by Equations 17 and 25 .

In summary, the target calculation for the distributed system will try the feasible routine first, and if it fails to obtain the feasible solution, then it will try the infeasible routine. The procedure requires a number of communications that are listed below:

1. (optional) A local controller initializes a setpoint change to the coordinator.

2. The coordinator receives new setpoints (from outside or local controller), update them to local controllers, and requests feasible response from local controllers.

3. Local controllers send feasible information to the coordinator, which includes $u_{p}^{i}$ and $V_{i}$.

4. After the coordinator confirms that it has received all the feasible information from local controllers, it conducts the optimization of Equation 22.

5. If feasible solution $\alpha^{*}$ is obtained, then goto step 8. Otherwise request infeasible response from local controllers.

6. Local controllers send infeasible information to the coordinator, which includes $u_{p}^{i}, V_{i}$, $z_{i}$, and $C_{i} V_{x, i}$.

7. After the coordinator confirms that it received all the infeasible information from local controllers, conduct the optimization of Equation 31 or 33 and get the optimal $\alpha^{*}$.

8. Update optimal $\alpha_{i}^{*}$ to local controllers.

9. Local controllers construct their local optimized inputs and states by using Equations 17 and 25.

Dimension Analysis. For the centralized constraints in this approach, the dimension of $\alpha$ equals to original degrees of freedom plus $\sum_{k=1}^{n_{u}} n_{u}^{k}$. However, the equality constraints introduced above have canceled out the extra dimensions and thus the degree of freedom remains the same. Also, the dimension of decision variables in this optimization problem is greatly reduced compared to optimization by using the centralized model.

\section{Case Study}

This paper has presented a coordination structure for distributed control, which provides an efficient way for communication, construction of distributed control, and calculation of target tracking. This section provides a case study on Tennessee Eastman (TE) challenge problem [23] to illustrate the efficiency of the proposed method, especially for the distributed target tracking calculation. 


\subsection{Distributed Control}

The main objective in the TE problem is to maintain the product flowrate and composition in spite of various faults (step changes, drifts and increase in noise levels) [23]. The process contains three main operation units: a reactor, a separator, and a stripper. It has 41 measured variables and 12 manipulated variables (MV), four reactants labeled as A, C, $\mathrm{D}$, and $\mathrm{E}$, two products labeled as $\mathrm{G}$ and $\mathrm{H}$. The construction of the distributed control is briefly stated here.

Since the product concentration is directly related to control objective, the concentration of $\mathrm{G}$ in the product and the product flowrate are chosen as the controlled variables (CV). Also, the reactor pressure is very sensitive to changes in process operation, which may cause safety issues. Hence, reactor pressure is considered as another controlled variable. For the MVs, several factors affect the concentration of $\mathrm{G}$ in the product. The feed $\mathrm{D}$ is the material that forms product $\mathrm{G}$, and $\mathrm{E}$ forms product $\mathrm{H}$ and thus will also influence the ratio of $\mathrm{G}$. Therefore, flowrates of $\mathrm{D}$ and $\mathrm{E}$ feed are chosen as the two MVs. Moreover, the reactor pressure control is challenging because it is too sensitive to several variables, including $\mathrm{D}$ feed and $\mathrm{E}$ feed. So when we conduct the concentration control and changes in $\mathrm{D}$ and $\mathrm{E}$ feeds, the reactor pressure will be affected significantly. Initially, the purge flowrate was picked as the MV for regulating the reactor pressure, however two problems arose. First, the dynamic between purge flowrate and reactor pressure is not fast enough to cover dramatic changes in reactor pressure. Secondly, the range of purge flowrate variation cannot compensate very large changes in reactor pressure. Thus another MV is added to help reactor pressure control, and the reactor temperature is chosen as MV. The response of reactor pressure with respect to reactor temperature is fast, but the drawback is that a large temperature change could cause instability. This possibility should be monitored and dealt with during control.

For distributed control, the system is partitioned to 2 subsystems according to their sampling rates. The reason is that subspace identification yields poor prediction if all the CVs are sampled at fast rate, while applying zero-order hold on the controlled variable with slower sampling rate. Then as shown in Figure 3, product flowrate and reactor pressure are placed in subsystem 1 , and $\mathrm{G}$ concentration in product is placed in subsystem 2 . The MVs are also classified: $\mathrm{D}$ feed is in subsystem 2 because it is the main MV that controls $\mathrm{G}$ concentration in product, and the other $3 \mathrm{MVs}$ are in subsystem 1.

The system model is obtained by subspace identification. Simulation results indicate that the reactor temperature and purge flowrate have little influence on the concentration of $\mathrm{G}$ in the product stream, therefore they are not considered as inputs to subsystem 2 . This could provide the advantage of eliminating the irrelevant pairs of $\mathrm{CV}$ and $\mathrm{MV}$ in a system, thus avoiding ill-conditioning during identification and improve model accuracy. Pseudo random ternary sequence (PRTS) signals are sent to MVs to stimulate the system to generate data rich in dynamic variations for open-loop system identification. The amplitudes of the PRTS are listed in Table 1.

A modification of distributed control for multi-rate sampled system under the proposed coordination scheme is utilized to control TE process. We developed a new approach to solve the control problem of multi-rate sampled systems that utilizes the lifted model, and 


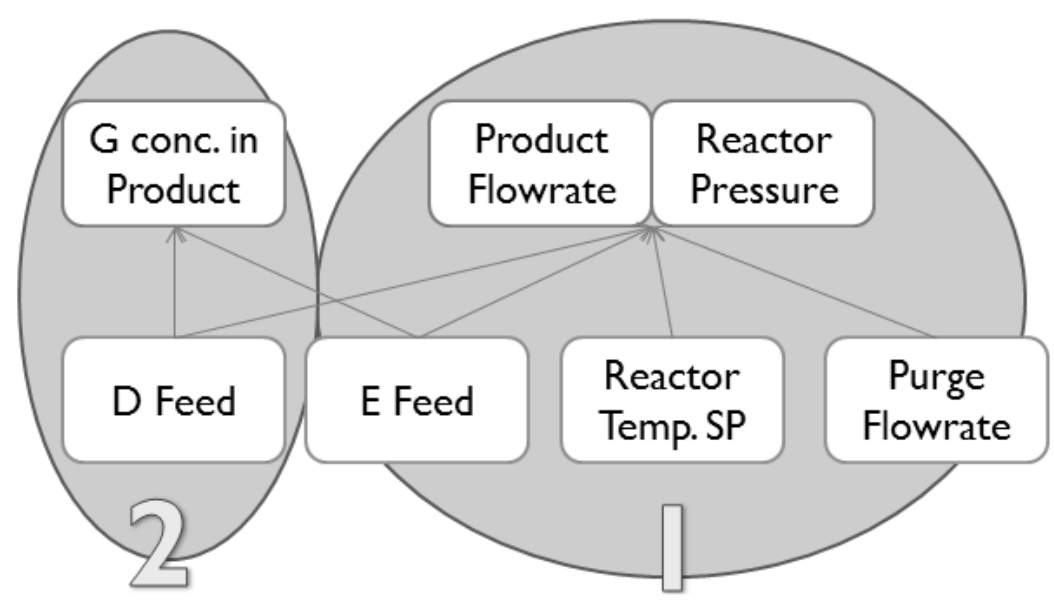

Figure 3: Distributed system partition.

Table 1: Notations of MVs for TE process, and the amplitudes of PRTS and step changes for system identification.

\begin{tabular}{ccc}
\hline \hline Notation & MV & $\delta u$ \\
\hline$u_{1}$ & D feed & $200 \mathrm{~kg} / \mathrm{s}$ \\
$u_{2}$ & E feed & $200 \mathrm{~kg} / \mathrm{h}$ \\
$u_{3}$ & Reactor temp. & $0.5{ }^{\circ} \mathrm{C}$ \\
$u_{4}$ & Purge flowrate & $0.05 \mathrm{kscmh}$ \\
\hline
\end{tabular}

leverages the advantages of distributed control techniques. In the proposed method, system outputs are assigned to different subsystems based on their sampling times (in each subsystem, all the controlled variables have the same sampling time, but it is not necessary to have all the variables that have the same sampling time in a single subsystem). Then, only input lifting (to basic rate) is required when identifying the lifted model of one subsystem, with the outputs unchanged, which further reduces the dimensions of the subsystem models. For the model of a subsystem, the influences from neighbor subsystems also need to be considered in order to develop distributed control. Thus, system identification should also include the related inputs of neighbor subsystems as inputs to this subsystem. Subspace identification is utilized to obtain the state space model for each subsystem. After the distributed models under different sampling times are available, DMPC is proposed to design the control system for the process with multi-rate sampling. The details of multi-rate distributed control is addressed in another paper [24], however the whole framework and procedure is unchanged, and thus further demonstrates the flexibility of this method. The control objective in this case is to reduce the product $\mathrm{G}$ concentration by $5 \%$, and increase the reactor pressure by 50 $k P a$. The control results are shown in Figure 4, which indicates successful target tracking for a setpoint change near the operation point. 

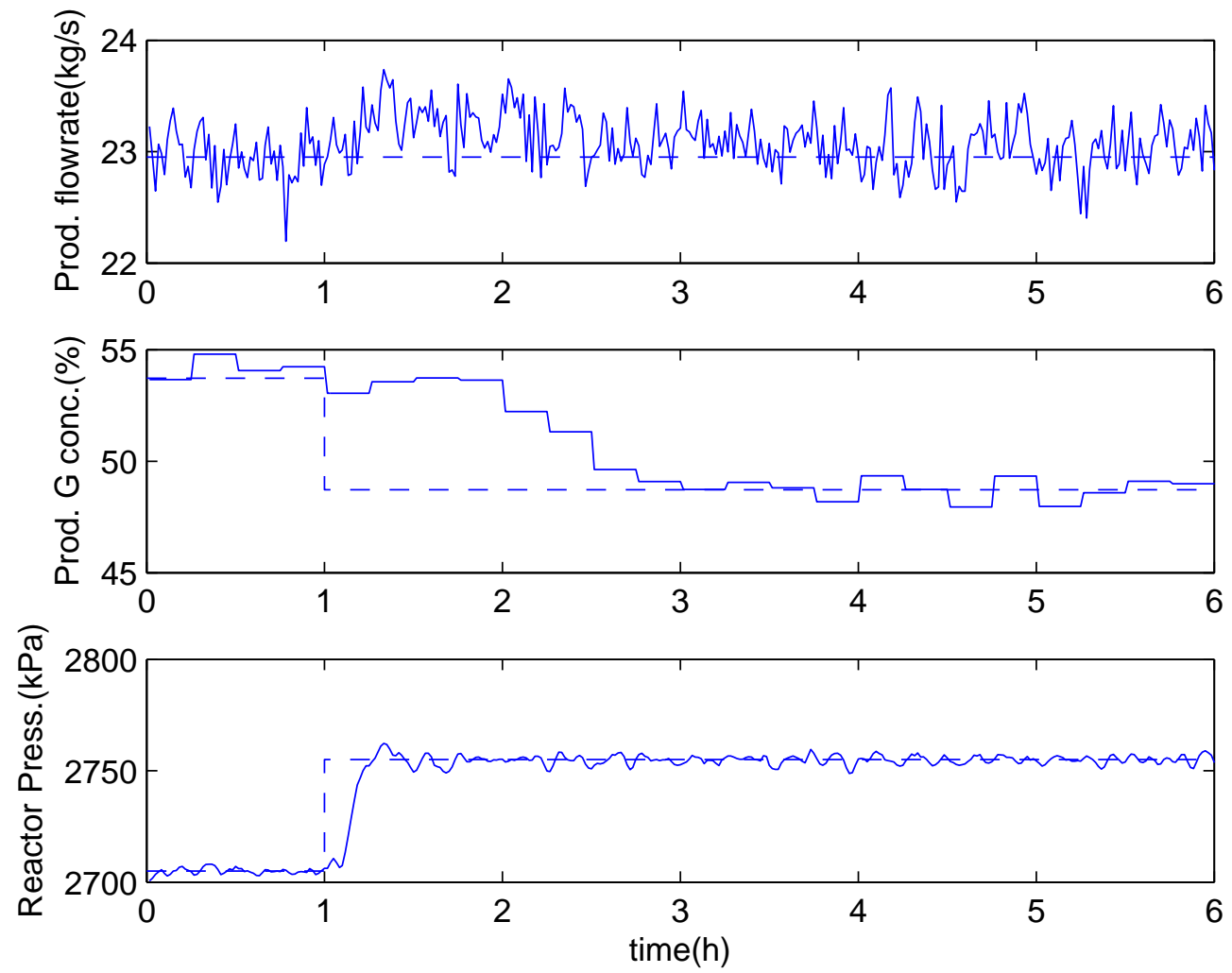

Figure 4: Distributed control for target tracking, the setpoint of product $\mathrm{G}$ concentration is reduced 5\%, and the setpoint of the reactor pressure is increased $50 \mathrm{kPa}$. The solid line shows the measurements of the CVs, and the dashed line shows the setpoints.

\subsection{Calculation of Target Tracking}

Lastly, the proposed target tracking calculation could reduce computation time compared to the setpoint calculated by constructing a single overall model, without the necessity to have a feasible starting point. The complexity and computational load in this approach are very favorable. The convex quadratic programming can be solved in polynomial time [25], and some algorithm could have the complexity of $\mathbf{O}\left(n^{3} L\right)$, where $L$ is the defined input size [26]. For the simpler linear algebra computation, the linear equation solving needs less than $\mathbf{O}\left(n^{3}\right)$ arithmetic operations, and finding null space by QR decomposition needs $\mathbf{O}\left(n^{3}\right)$ arithmetic operations.

In the TE problem, the identified model for subsystem 1 has 15 states, 2 outputs, 3 local inputs, and 1 neighbor input; the identified model for subsystem 2 has 12 states, 1 output, 1 local input, and 1 neighbor input. In case the centralized optimization is conducted to calculate target tracking, the centralized model will have a total of 27 states, 3 outputs, and 4 inputs, therefore it has 31 decision variables (number of states plus number of inputs) and requires 30 equality constraints (number of states plus number of outputs).

In the proposed approach, each subsystem conducts linear equation solving for particular 
solution, plus the calculation for the null space of $A_{E}$. On the coordinator side, the quadratic optimizations for target tracking has 3 decision variables and 2 equality constraints. Moreover, the computation of general solutions in local controllers could be done in parallel, thus the total computational time should equal to the $\max \left(t_{1}, t_{2}\right)+t_{c}$, where $t_{i}$ is the time spent on finding the general solution in subsystem $i$, and $t_{c}$ is the time spent on the coordinator.

Two cases are studied for the target tracking calculation for comparison between the method based on a single overall model and the proposed method. The two cases are to set the $\mathrm{G}$ concentration in product to $75 \%$ and $90 \%$ separately. Case 1 (75\%) is a moderate condition, while Case $2(90 \%)$ is infeasible in our setting. The matrices for each steps are extracted and written in Matlab files, and quadprog in Optimization Toolbox is utilized for quadratic optimization. Each case has run 100 times for each method, and they generated identical results. The average computation time is calculated and given in Table 2. The results show that the time saved is not as significant as it appears in the dimension deduction in the moderate situation. One reason is that the proposed coordinated method introduces inequality constraints to the quadratic programming, while the centralized method only has lower and upper bounds. However, in the case that no feasible solution is available (thus need to go to the infeasible routine), the proposed method spent much less time to figure out the infeasibility by leveraging the advantage of small number of decision variables (Case 2).

Table 2: Average calculation time (in $m s$ ) of target tracking.

\begin{tabular}{c|ccc|cc}
\hline \hline & $t_{1}$ & $t_{2}$ & $t_{c}$ & Coordinated & Centralized \\
\hline Case 1 & 0.257 & 0.203 & 1.947 & 2.204 & 3.628 \\
Case 2 & 0.256 & 0.202 & 1.758 & 2.014 & 12.522 \\
\hline
\end{tabular}

\section{Conclusions}

A coordination structure for distributed control is proposed, to construct the distributed control problem in a flexible way. This approach enables the calculation of the new steady states in target tracking in a distributed manner, with minimum calculation in the coordinator and without the requirement for a feasible starting point. The key concept behind this coordination scheme is to let the local controllers have high level of autonomy, thus each controller identifies its own model, stores its own information, and computes and implements its own control actions, with the coordinator playing an assistant role.

Acknowledgments - Partial support of the research conducted by National Science Foundation Grant CTS-0325378 of the ITR program is gratefully acknowledged.

\section{References}

[1] A. N. Venkat, J. B. Rawlings, S. J. Wright, Stability and optimality of distributed model predictive control, in: Decision and Control, 2005 and 2005 European Control Conference. CDC-ECC '05. 44th IEEE Conference on, 2005, pp. 6680-6685. 
[2] B. T. Stewart, A. N. Venkat, J. B. Rawlings, S. J. Wright, G. Pannocchia, Cooperative distributed model predictive control, Systems \& Control Letters 59 (8) (2010) $460-469$.

[3] J. Liu, D. Muñoz de la Peña, P. D. Christofides, Distributed model predictive control of nonlinear process systems, AIChE Journal 55 (5) (2009) 1171-1184.

[4] P. D. Christofides, R. Scattolini, D. Muñoz de la Peña, J. Liu, Distributed model predictive control: A tutorial review and future research directions, Computers \& Chemical Engineering 51 (2013) 21-41.

[5] J. B. Rawlings, B. T. Stewart, Coordinating multiple optimization-based controllers: New opportunities and challenges, Journal of Process Control 18 (9) (2008) $839-845$.

[6] B. T. Stewart, S. J. Wright, J. B. Rawlings, Cooperative distributed model predictive control for nonlinear systems, Journal of Process Control 21 (5) (2011) 698 - 704, special Issue on Hierarchical and Distributed Model Predictive Control.

[7] J. Liu, X. Chen, D. Muñoz de la Peña, P. D. Christofides, Sequential and iterative architectures for distributed model predictive control of nonlinear process systems, AIChE Journal 56 (8) (2010) 2137 2149.

[8] P. D. Christofides, J. Liu, D. M. De La Pena, Networked and distributed predictive control: Methods and nonlinear process network applications, Springer, 2011.

[9] M. Heidarinejad, J. Liu, D. M. noz de la Peña, J. F. Davis, P. D. Christofides, Multirate lyapunov-based distributed model predictive control of nonlinear uncertain systems, Journal of Process Control 21 (9) (2011) $1231-1242$.

[10] Y. Sun, N. H. El-Farra, Quasi-decentralized model-based networked control of process systems, Computers \& Chemical Engineering 32 (9) (2008) 2016 - 2029, networked and Complex Systems S.I. Control of Networked and Complex Process Systems.

[11] E. Camponogara, D. Jia, B. H. Krogh, S. Talukdar, Distributed model predictive control, Control Systems, IEEE 22 (1) (2002) 44-52.

[12] L. Magni, R. Scattolini, Stabilizing decentralized model predictive control of nonlinear systems, Automatica 42 (7) (2006) 1231-1236.

[13] A. Alessio, D. Barcelli, A. Bemporad, Decentralized model predictive control of dynamically coupled linear systems, Journal of Process Control 21 (5) (2011) 705 - 714, special Issue on Hierarchical and Distributed Model Predictive Control.

[14] M. D. Doan, T. Keviczky, B. D. Schutter, An iterative scheme for distributed model predictive control using fenchel's duality, Journal of Process Control 21 (5) (2011) 746 - 755, special Issue on Hierarchical and Distributed Model Predictive Control.

[15] A. Ferramosca, D. Limon, I. Alvarado, E. Camacho, Cooperative distributed $\{\mathrm{MPC}\}$ for tracking, Automatica 49 (4) (2013) 906 - 914.

[16] R. Scattolini, Architectures for distributed and hierarchical model predictive control c a review, Journal of Process Control 19 (5) (2009) $723-731$.

[17] E. Tatara, F. Teymour, A. Cinar, Control of complex distributed systems with distributed intelligent agents, J Process Control 17 (5) (2007) 415-427.

[18] S. Perk, F. Teymour, A. Cinar, Statistical monitoring of complex chemical processes using agent-based systems, Industrial and Engineering Chemistry Research 49 (2011) 5080-5093.

[19] A. Artel, F. Teymour, M. North, A. Cinar, A multi-agent approach using perceptron-based learning for robust operation of distributed chemical reactors, Engng Applications of Artificial Intelligence 24 (6) (2011) 1035-1045.

[20] S. Perk, F. Teymour, A. Cinar, An adaptive agent-based system for process fault classification and diagnosis, Industrial and Engineering Chemistry Research 50 (15) (2011) 9138-9155.

[21] S. Perk, Q. M. Shao, F. Teymour, A. Cinar, An adaptive fault-tolerant control framework with agentbased systems, International Journal of Robust and Nonlinear Control 22 (2012) 43-67.

[22] A. N. Venkat, J. B. Rawlings, S. J. Wright, Stability and optimality of distributed, linear model predictive control part i: state feedback, Tech. rep., University of Wisconsin, Madison (2006).

[23] J. J. Downs, E. F. Vogel, A plant-wide industrial process control problem, Computers \& Chemical Engineering 17 (3) (1993) 245-255. 
[24] Q. M. Shao, A. Cinar, System identification and distributed control for multi-rate sampled systems, manuscript submitted (2014).

[25] M. K. Kozlov, S. P. Tarasov, L. G. Khachiyan, The polynomial solvability of convex quadratic programming, USSR Computational Mathematics and Mathematical Physics 20 (5) (1980) 35-45.

[26] R. Monteiro, I. Adler, Interior path following primal-dual algorithms. part ii: Convex quadratic programming, Mathematical Programming 44 (1989) 43-66. 